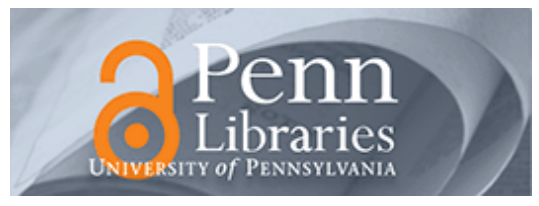

University of Pennsylvania

ScholarlyCommons

Marketing Papers

Wharton Faculty Research

1984

\title{
Conjoint Analysis of Price Premiums for Hotel Amenities
}

Stephen M. Goldberg

Paul E. Green

University of Pennsylvania

Jerry Yoram Wind

University of Pennsylvania

Follow this and additional works at: https://repository.upenn.edu/marketing_papers

Part of the Advertising and Promotion Management Commons, Business Administration,

Management, and Operations Commons, Hospitality Administration and Management Commons, and the Marketing Commons

\section{Recommended Citation}

Goldberg, S. M., Green, P. E., \& Wind, J. (1984). Conjoint Analysis of Price Premiums for Hotel Amenities. Journal of Business, 57 (1), S111-S132. Retrieved from https://repository.upenn.edu/marketing_papers/ 398

This paper is posted at ScholarlyCommons. https://repository.upenn.edu/marketing_papers/398

For more information, please contact repository@pobox.upenn.edu. 


\title{
Conjoint Analysis of Price Premiums for Hotel Amenities
}

\author{
Abstract \\ Metric hybrid conjoint models have received a fair amount of industry application to date. The purpose of \\ these models is to reduce data collection time while still retaining individual differences in part-worth \\ functions. The present paper extends this class of models to include categorical conjoint analysis in \\ which the criterion variable is classificatory. This model is applied to an extremely large conjoint problem \\ involving over 40 attributes and over 100 attribute levels. The study results support the viability of the \\ model for dealing with extremely large conjoint problems. The study also shows evidence of the inability \\ of simple functions of self-explicated utilities for components of a bundle of hotel amenities to predict \\ respondents' preferences for the total bundle.

\section{Disciplines} \\ Advertising and Promotion Management | Business | Business Administration, Management, and \\ Operations | Hospitality Administration and Management | Marketing
}




\section{Introduction}

Over the past decade or so, conjoint analysis (Green and Rao 1971; Johnson 1974) appears to have become one of industry's most widely applied marketing research techniques for estimating consumer's multi-attribute utility functions (Cattin and Wittink 1982). However, a continuing problem in application of conjoint analysis is how to handle the price attribute in the construction of stimulus profiles for presentation to respondents. In many cases of practical interest, the price level will be highly correlated with other product attribute levels. If the researcher ignores this correlation by allowing the price levels to vary independently of other attributes, the resulting profiles may appear sufficiently unrealistic to result in respondents' discounting each profile's attractiveness, thus reducing the validity of the results.

A related problem which adds to the complexity of new product pricing research is the bundling issue. In the context of conjoint analysis, the question is whether one can predict a respondent's evaluation of a bundle of product attributes and price as a simple linear function of the summed part worths of the entities making up the bundle when each entity is presented with its actual price.

(Journal of Business, 1984, vol. 57, no. 1, pt. 2)

(C) 1984 by The University of Chicago. All rights reserved. $0021-9398 / 84 / 5712-0019 \$ 01.50$
Metric hybrid conjoint models have received a fair amount of industry application to date. The purpose of these models is to reduce data collection time while still retaining individual differences in part-worth functions. The present paper extends this class of models to include categorical conjoint analysis in which the criterion variable is classificatory. This model is applied to an extremely large conjoint problem involving over 40 attributes and over 100 attribute levels. The study results support the viability of the model for dealing with extremely large conjoint problems. The study also shows evidence of the inability of simple functions of self-explicated utilities for components of a bundle of hotel amenities to predict respondents' preferences for the total bundle. 
An excellent example of both the correlated attributes and bundling problems involves the pricing of hotel amenities, such as king-sized beds, built-in bar facilities, in-room movies, and the like. Respondents (rightly) believe that these enhancements will carry some kind of premium over the basic price of the room. The price premium might be expressed on an individual amenity basis or, more likely, as an extra cost for a complete bundle of amenities.

This paper is concerned with ways in which conjoint methods may be modified to deal with the correlated attributes and bundling problems. In the first two sections we briefly describe some of the ways in which correlated attributes (such as price, vis-à-vis other product attribute levels) and bundling (expressing a price premium for each amenity and also for complete bundles of amenities) can be handled. We then outline the research questions which guided this study and propose a hybrid model employing categorical conjoint analysis for dealing with the large number of attributes and levels that characterize a hotel offering.

The model is applied to actual data on lodging preferences, recently collected by a large hotel chain. We show how the categorical conjoint model provides an efficient way to estimate utilities for large numbers of attribute levels while still retaining individual differences. The paper concludes with a discussion of the empirical implications of the study as related to measuring utilities for individual enhancements versus the cumulative impact of a bundle of enhancements (and its associated price) on respondent preferences.

\section{Correlated Attributes}

The effect of correlated attribute levels on a subject's preference evaluations of experimental stimuli has been of concern to researchers in the multi-attribute utility measurement area as well as to researchers interested in cue consistency and to market researchers. In the multiattribute utility modeling area (Edwards 1976; Einhorn and McCoach 1977), the presence of negative correlations (if recognized by the respondent) tends to increase the sensitivity of the criterion function (e.g., preference) to the choice of appropriate attribute importance weights. This situation is an important exception to the prevailing use of simplified attribute weighting schemes, such as unit weights (Dawes and Corrigan 1974), which work nicely in the case of positively correlated attributes.

In the cue consistency area, investigators of "policy capturing" modeling (Anderson and Jacobson 1965; Dudycha and Naylor 1966) have found that the lack of cue consistency (i.e., failure to observe environmental correlations) tends to reduce the importance of the affected attributes. Similarly, Slovic (1966) found experimental evidence that when "inconsistent" profiles are employed, subjects tend to rely 
on only one of the two cues, the selected cue depending on its perceived relationship with the other cues in the profile. In a similar experiment, Hoffman (1968) found that a cue will be strongly weighted if it has a high validity with the criterion but will decline in importance if perceived to be incongruent with other profile cues.

In the context of conjoint analysis, Green and Srinivasan (1978) discuss the problem of correlated attributes. For studies using the full profile data collection method, three general approaches to the problem have been described:

1. If the environmental correlations are low, the researcher may wish to assume complete independence and take advantage of highly efficient partial factorial designs (Green, Carroll, and Carmone 1978), such as main effects plans, compromise designs, or resolution IV and $\mathrm{V}$ designs (Addelman 1962). The virtue of these designs is their high efficiency and unambiguous allocation of accounted-for variance to each contributing attribute.

2. If some subset of attributes is highly correlated environmentally (e.g., automobile acceleration, gas mileage, horsepower, and top speed), the researcher can prepare a composite attribute, each level of which represents a cluster of the more basic attribute levels. As such, it is no longer possible to separate out the effects on utility of the subfactors making up the composite factor.

3. In the intermediate case of moderate environmental attribute correlations, the researcher may wish to sample attribute levels from some multivariate distribution that reflects these correlations (Parker and Srinivasan 1976). In the process one might try to make the attribute correlations in the resultant sample of stimulus profiles somewhat lower than those in the environment in order to increase the statistical efficiency of the estimates and reduce ambiguity in the allocation of shared variance across the set of attribute levels.

Translated into the case of hotel amenity pricing, the preceding comments suggest that the researcher could construct stimulus profiles in which $(a)$ the price varies independently of the amenity; $(b)$ the price depends completely on the amenities (and hence their separate part worths cannot be determined in the analysis); or (c) the price is highly (but not perfectly) correlated with the type of amenity. This third alternative can be implemented by including an experimentally designed deviation term to be added algebraically to the "true" price given a specific amenity. The larger the deviation term relative to the true price, the less the correlation but the lower the profile's realism.

\section{Bundling}

Recent years have witnessed the growth of an economic literature on bundling (Stigler 1968; Adams and Yellen 1976; Telser 1979; Spence 1980; Paroush and Peles 1981; Phillips 1981; Schmalensee 1984). The 
focus of this literature is on the use of bundling - package selling - as a price discrimination device and its normative effects on seller profits, consumer's surplus, and net welfare. Three bundling strategies are typically identified-unbundled sales (pure component strategy), pure bundling (only the bundle is offered), and mixed bundling (offering both a bundle and individual components) (Adams and Yellen 1976).

Despite the conceptual attractiveness and elegance of this research, little attention has been given to the empirical study of consumers' preferences for bundled versus unbundled products and the researcher's ability to predict preferences for a bundle of product attributes and price combinations from consumers' evaluations of the entities making up the bundle.

This problem was described in a succinct and lucid way by Tversky (1969). Assume that a person is about to purchase a new compact automobile. His initial desire is to choose the cheapest, stripped-down model. However, when the salesperson points out that air conditioning can be had for only $\$ 375$ over the base price, he feels that the additional cost is more than outweighed by the anticipated comfort. Similar reactions are expressed in the case of other extra-cost options, such as power steering, power brakes, and an AM-FM radio, when each of these is presented one at a time, with its associated cost. Only when the cost of these options is added up does the buyer realize that he really prefers the stripped-down version to the loaded model.

In the context of hotel amenity pricing, our substantive interest is in finding out if one can predict a respondent's evaluation of a bundle of hotel amenity-price combinations as a simple linear function of the summed part worths of the entities making up the bundle. Prices are presented in two ways - the incremental price (premium over the basic price of a room) for each of the entities and the total room price for each of the full-profile bundles of hotel amenities.

\section{Research Questions}

The substantive research questions of interest are: (1) Can one's preference for a bundle of hotel amenities (and their overall price) be predicted as a simple (e.g., linear) function of the respondent's part worths for the bundle components? (2) If not, does the overall bundle price account for significant variance beyond that associated with the bundle's components? (3) If so, what is the shape of the pricepreference function? (4) How well can one predict each respondent's bundle preferences, based on parameters estimated from the rest of the sample?

From a methodological viewpoint there are two questions of principal interest in this study: (1) Does a hybrid model, based on categorical conjoint analysis, produce "reasonable" results? (2) Do respondentobtained importance weights provide greater predictive validity than simple (i.e., equal) weights? 


\section{The Model}

The research design developed to answer these research questions centered on a hybrid categorical conjoint analysis model.

\section{Hybrid Models}

Hybrid conjoint models have been developed recently to cope with a practical problem in applied conjoint analysis, namely, the need to streamline the data collection task while still preserving some individual differences in utility functions (Green, Goldberg, and Montemayor 1981; Green, Goldberg, and Wiley 1982). Hybrid conjoint models adapt an old idea-self-explicated utility assessment (Wilkie and Pessemier 1973) - to conjoint analysis. While a number of hybrid models have been proposed, each procedure entails the consideration of some type of self-explicated utility where respondents evaluate the levels of each attribute (one attribute at a time) on some type of desirability scale. This is followed by an evaluation of the attributes themselves on an importance scale.

A respondent's self-explicated utility for the $h$ th stimulus profile is usually assumed to be given by a simple additive model,

$$
U_{h}=\sum_{j=1}^{J} w_{j} u_{i_{j}}^{h}
$$

where $U_{h}$ is the total utility of alternative $h, w_{j}$ is the self-explicated importance weight of attribute $j$, and $u_{i j}^{h}$, denotes the fact that alternative $h$ has a desirability score of $u$ on level $i$ of attribute $j$. (For ease of presentation the respondent index is suppressed.)

The next stage of the data collection involves presenting each respondent with a limited set (usually eight or nine) of complete (allattribute) stimulus profiles. These stimulus profiles, in turn, are drawn from a much larger master design (usually ranging between 64 and 256 profiles) that permits orthogonal estimation of all main effects and selected two-way interactions. Moreover, profiles can be "balanced" within respondent by means of various blocking designs. The respondent then evaluates each complete stimulus profile on some type of likelihood of purchase or intentions-to-buy scale. Call each of these responses $Y_{h}$.

The matrix $\mathbf{S}$ of utility functions, of order $N$ by $\sum_{j=1}^{J} I_{j}$ for the $N$ respondents, as obtained from the self-explicated task of stage 1, is row centered and/or standardized to zero mean and unit standard deviation. That is, each respondent's specific set of $w_{j} u_{i}$ 's in equation (1)-there are $\Sigma_{j=1}^{J} I_{j}$ of these for each respondent-are often expressed as deviations from his or her mean. Respondents are then clustered on the basis of similarities in their self-explicated utility functions. Assume that $k$ clusters are found. 
The hybrid model's parameters are then separately estimated for each cluster by means of OLS regression. The hybrid model is defined as follows:

$$
\begin{aligned}
Y_{h} & =Y_{i_{1} i_{2}} \cdots i_{J} \\
& \cong a+b U_{i_{1} i_{2}} \cdots i_{J}+\sum_{j=1}^{J} v_{i_{j}}+\sum_{j<j^{\prime}} t_{i j^{\prime}}
\end{aligned}
$$

where each $U_{i_{1} i_{2}} \ldots i_{j}$ is separately computed for each respondent via equation (1); $a$ is an intercept term, $b$ is a regression parameter representing the contribution of the self-explicated utility to $Y$, and the $v$ 's and $t$ 's are also regression parameters, estimated at the cluster level. The $v$ 's denote main effects while the $t$ 's denote selected two-way interactions.

Metric hybrid models, of the type illustrated above, have been applied to a variety of industrial applications and appear to work well in the more typical case of 7-10 attributes. A nonmetric version of the model (utilizing dummy variables for combinations of desirability and importance) has received some limited application (Green and Goldberg 1981). However, neither of these models is designed to deal with the problem illustrated by the hotel amenity pricing case.

\section{A Hybrid Categorical Conjoint Analysis Model}

Categorical conjoint analysis (Carroll 1969), in contrast to ordinal methods like MONANOva (Kruskal 1965) and LINMAP (Shocker and Srinivasan 1977), has received relatively little application to date. In categorical conjoint analysis the analyst does not necessarily have to assume that the dependent variable (e.g., most preferred, acceptable, unacceptable) is ordered. Rather, the basic idea is to find scale values associated with the independent variables (expressed as dummies) such that additive combinations of these scale values maximally correlate with similarly obtained scale values for the response categories.

As Carroll points out (Carroll 1973), if the categories of the dependent (i.e., response) variable precisely maintain the hypothesized rank order, then the analysis is precisely equivalent to MONANOva. In general, however, there will be some departures, if only due to noise in the data. However, if departures from monotonicity of the dependent varible are large and systematic, the assumed underlying order of the response categories may be called into question.

From a practical viewpoint categorical conjoint analysis can be implemented by means of a dummy variable canonical correlation program. In the present application, we can make the dummy variable 
assignments to phase 1 responses shown in the unnumbered table below.

\begin{tabular}{|c|c|c|}
\hline & & $Y_{1} Y_{2}$ \\
\hline Most preferred level & $\Rightarrow$ & 10 \\
\hline Acceptable level & $\Rightarrow$ & 01 \\
\hline Unacceptable level & $\Rightarrow$ & $\begin{array}{ll}0 & 0\end{array}$ \\
\hline
\end{tabular}

The predictor variables will also consist of dummies $-I_{j}-1$ dummy variables for each set of $I_{j}$ attribute levels. For example, in the facet dealing with associated hotel services (fig. 1), the first attribute is message service, with four levels. These levels can be coded into three dummies as shown below.

\begin{tabular}{|c|c|c|}
\hline & & $X_{1} X_{2} X_{3}$ \\
\hline Pick up note at front desk & $\Rightarrow$ & $\begin{array}{lll}0 & 0 & 0\end{array}$ \\
\hline Light on phone & $\Rightarrow$ & 100 \\
\hline Light on phone and note under door & $\Rightarrow$ & $\begin{array}{lll}0 & 1 & 0\end{array}$ \\
\hline Recorded voice of sender & $\Rightarrow$ & $\begin{array}{lll}0 & 0 & 1\end{array}$ \\
\hline
\end{tabular}

As noted, for each attribute $j$ with $I_{j}$ levels, there are $I_{j}-1$ dummy variables. Attributes not occurring during any given evaluation are coded zero. Hence, in the case of associated services (fig. 1), there are two dummy criterion variables and 28 dummy predictor variables.

Since we wish to make comparisons among all attribute levels within a given facet (such as associated services), we employ a covariance matrix rather than correlation matrix in computation of the canonical regression weights (i.e., part worths). Since each part-worth function is a separate interval scale with common unit but arbitrary origin, we are free to set the lowest part worth, within attribute, at zero. Furthermore, so as to compare part worths across facets, we will normalize the results within facet so that the maximum utility range is set to vary from 0 to 10 .

In sum, we will use categorical conjoint analysis to find part worths for the attribute levels in each facet. Within facet, these are analogous to the $u_{i}$ 's of equation (1). Next we will employ the respondentobtained facet importances (from the constant sum task of phase 2) as weights that are analogous to the $w_{j}$ values of equation (1). Hence, for each complete profile in phase 3 , we have an initial prediction based on the equation

$$
Y_{i_{1} i_{2}} \ldots i_{J^{*}} \cong a+b U_{i_{1} i_{2}} \ldots i_{j^{*}}
$$

where $J^{*}$ now denotes the total number of attributes across all facets. If the above linear function of the self-explicated utilities is sufficient to predict the $Y$ responses (in phase 3), then we will not need to introduce 


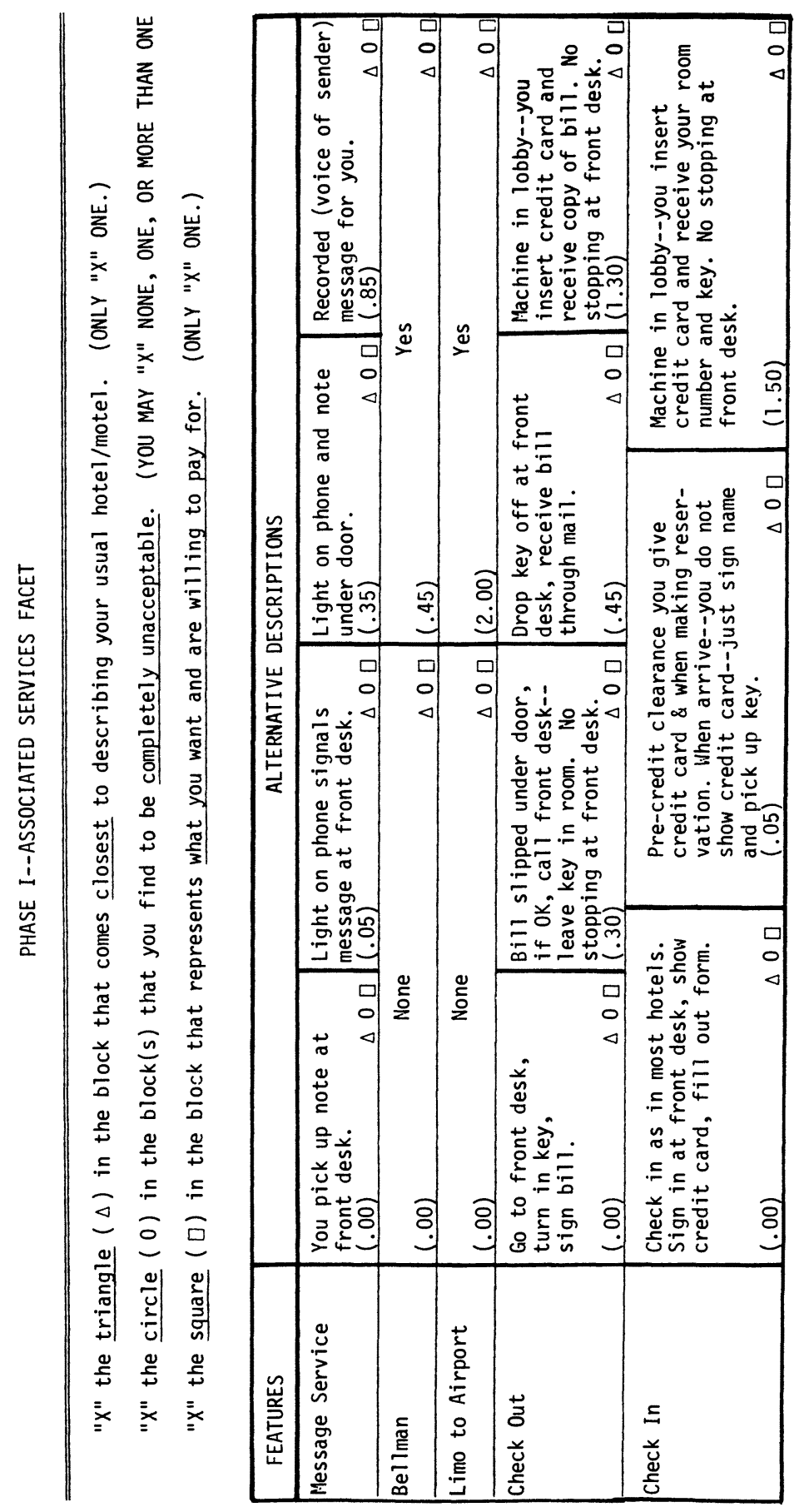




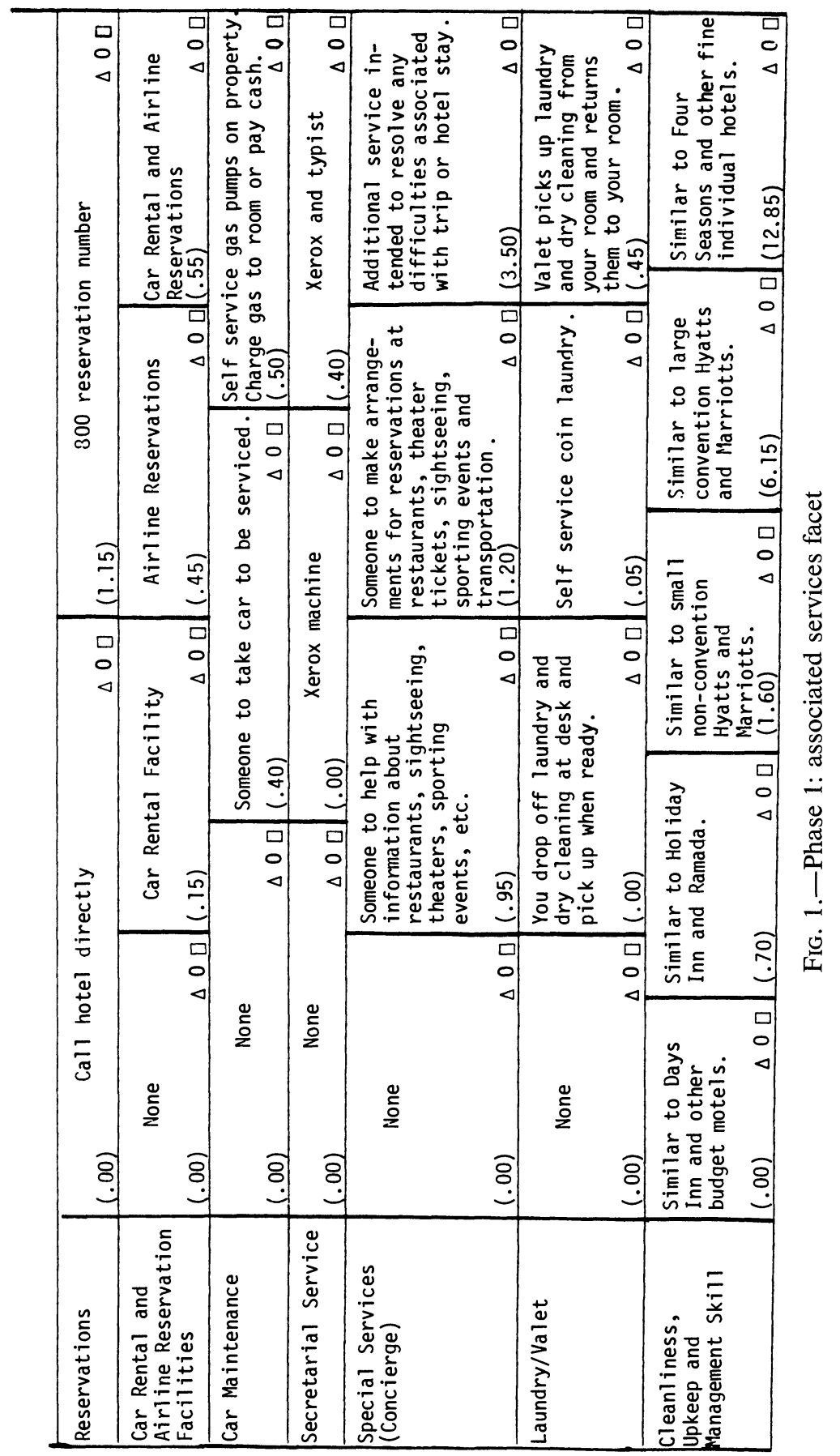


the total price variable (obtained by summing the separate premiums and adding this total to the basic room price).

\section{The Empirical Study}

Data for the analysis were collected by professional interviewers. The sample employed in this analysis consisted of 180 adults, all of whom had lodged at least one night (for business purposes) within a 6-month period prior to being contacted for the survey. ${ }^{1}$ Respondents were recruited by telephone screening in nine standard metropolitan statistical areas scattered over the United States. All interviews were personally administered in central facilities; respondents received a small gift for their participation. ${ }^{2}$

\section{Stimulus Materials and Data Collection}

The data collection task consisted of three phases. After an introduction that emphasized interest in the respondent's preferences for hotel amenities related to business trips, each respondent received six cards, one at a time. Each card dealt with one facet of hotel facilities: atmosphere and physical facilities, room itself, associated hotel services, recreation/sports, lounge/entertainment, and security.

Figure 1 shows the card describing the "associated hotel services" facet. There are 12 features (i.e., attributes) with levels ranging from 2 to 5 each. Each profile was presented with its associated price. For example, in the case of message service, there are four levels, ranging from picking up notes at the front desk to obtaining a recorded voice of the sender's message. In each block a price appears in parentheses, ranging from (.00) for no premium for front desk message pickup to $(.85)$, or $\$ 0.85$ for the recorded message. The specific price levels were developed by the sponsor's cost accounting department, and no experimental price variations were introduced.

Respondents were asked to think about their usual hotel stay (for business purposes) and to check the triangle in each row that best described the hotel. (These data were not analyzed in the present paper.) Next the respondent supplied one of three possible answers to each amenity-price combination: whether the combination is completely unacceptable, whether the combination is most preferred, and whether the combination is acceptable (by implication). The procedure (adapted from a data collection method used by Rank-Xerox) was then repeated for the remaining five facets. Figure 2 lists the attribute descriptions and number of levels associated with the remaining five

1. Data were also collected on nonbusiness travelers; however, this analysis is not discussed here.

2. In accordance with the sponsor's wishes, all price data have been coded; however, relationships among the variables reflect those found in the analysis. 


\section{Atmosphere/Facilities}

Hotel Size

Corridor/View

Pool Location

Pool Type

Landscaping

Building Shape

\section{Room}

Heat/Cooling

Quality of Decor

Size

Rental Entertainment

Type of In-Room TV Bathroom Amenities

Bathtub Features

Sink Location

Bathtub Size

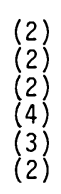

(4)

(5)
Recreation/Sports

Pool Extras

Children's Playroom (4)

Game Room

Tennis Courts

Racquetball Courts

Exercise Room (3)

Whirlpool/Jacuzzi (3)

Sauna

Lounge Entertainment

Nearby Lounge

Type of People

Atmosphere

(3)

(3)

Security

Alarm Button

24-Hour Viedo

Sprinkler System

Smoke Detector

Security Guard

(4)

FIG. 2.-Attribute descriptions and number of levels (in parentheses) of the remaining five facets.

facets. A total of 43 attributes across the six facets is involved. The number of attribute levels is well in excess of 100. (Visual aids were used, where appropriate, to describe the various attribute levels.)

In phase 2 respondents were shown a card listing the six facets whose detailed attribute levels had been evaluated earlier. In this case the respondents were asked to distribute 100 points across the six facets to reflect their relative importance in the choice of a hotel room for business purposes; this task entails a simple constant sum procedure.

In phase 3 respondents were shown five full-profile description cards one at a time, depicting a "complete" hotel offering. Each set of five descriptions was balanced within subject and drawn from a master partial factorial design of 50 profiles. In this case each of the six facets was treated as an experimental factor with five levels each; hence the $5^{6}$ full factorial $(15,625$ combinations) was represented by an orthogonal main effects plan, entailing less than $1 \%$ of the total number of combinations. (See fig. 3 for an illustration of one such full-profile card.)

With each facet, the five levels were constructed by the sponsor's 


\section{ATMOSPHERE/}

FACILITIES
- Small (125) rooms, 2-story hotel with enclosed central corridors and stairs

- All rooms have individual balcony or bay window

- Spacious outdoor courtyard with freeform pool and elaborately landscaped grounds

ROOM

- Typical size room with full control of central heating and cooling

- Quality of decor similar to Hyatt Regency

- Color TV, HBO movie channel, rental movies

- Large soap, bath gel, shower cap, shampoo, shower massage, separate sink

- Oversize bathtub with shower

\section{ASSOCIATED \\ SERVICES}

\section{RECREATION/}

SPORTS

- Children's playroom, pinball game room, exercise
room

\section{LOUNGE/}

ENTERTAINMENT - Nearby lounge, open to public

SECURITY

PRICE (SINGLE)
- Whirlpool, sauna

- Quiet Atmosphere

- 24-Hour video camera and security guard

- Smoke detector in room; sprinkler system for lobby and hallways

- Machine-assisted check-in and check-out

- Light-on-phone message system and valet

Similar in cleanliness to small, non-convention Hyatts

$\$ 53.75$

Consider the above description and room price (single room, inclusive of all options). Please circle the number below that best describes how likely you would be to stay. A "1" means you'd probably never stay there and a "10" means you'd be almost certain to stay there.

Probably

Would Never

Stay There
Almost Certain to Stay There 10 
personnel to "cover" the range of interest. As might be expected, attribute levels tended to be correlated within each facet so that premium-priced amenities often clustered together. However, across facets that orthogonality of the master design was respected. For each of the five phase 3 hotel descriptions that respondents received, they were asked to indicate the likelihood that they would stay there. Completion of phases 1 through 3 required about 35 minutes on the average. $^{3}$

\section{Analysis Outline}

The analytical steps followed in computing utility functions for the hotel amenity-price evaluations were:

1. For each of the six facets in phase 1 , a categorical conjoint analysis was made of that facet's response data. Each part worth was scaled so that the lowest utility in each facet was zero and the maximum range was 0-10.

2. Each respondent's self-explicated utility was computed (with individual importance weights) to obtain a predictor variable for phase 3 evaluations.

3. Parameters of the linear function of equation (3) were then computed.

4. Residuals from this step were found and regressed on the total room price (shown to the respondent in phase 3 ). We then determined whether the inclusion of this additional set of variables accounted for significant variance in the residuals.

The preceding steps constituted the main thrust of the analysis. Hcwever, these steps were augmented to consider such methodological questions as: How accurate are the results at the individual level? How sensitive are the predictions to changes in facet importance weights? What is the effect of the blocking variable (i.e., the particular five profiles out of 50 evaluated by the respondent in phase 3 )?

\section{Results}

Results of the analysis are presented on a summary basis, in terms of the specific analytical steps/questions described earlier. The conclusions of this analysis, as related to the specific research questions which motivated this research project, are briefly highlighted in the Discussion section.

\section{Canonical Correlation Results}

Table 1 shows some summary results for the canonical correlation analysis of the data from phase 1 . The first column shows the canonical

3. While a number of other kinds of data were also obtained, we limit our analysis to responses to the three phases just described. 
TABLE 1 Results of Canonical Correlation Phase 1 Data

\begin{tabular}{lccc}
\hline & & \multicolumn{2}{c}{$\begin{array}{c}\text { Scale Values } \\
\text { (Normalized) }\end{array}$} \\
\cline { 3 - 4 } Facet & $\begin{array}{c}\text { First } \\
\text { Canonical } \\
\text { Correlation }\end{array}$ & $\begin{array}{c}\text { Most } \\
\text { Preferred }\end{array}$ & Acceptable \\
\hline Atmosphere/facilities & .38 & 1.00 & .11 \\
Room & .40 & 1.00 & .09 \\
Associated services & .61 & 1.00 & .35 \\
Recreation/sports & .62 & 1.00 & .05 \\
Lounge & .29 & 1.00 & .32 \\
Security & .47 & 1.00 & .12 \\
\hline
\end{tabular}

correlation associated with the first pair of linear compounds. All of these canonical correlations were significant at the .001 level or better. From a practical standpoint the correlations are only modest; they range from .29 to .62 . Interestingly enough, canonical correlations for the second pair of linear compounds (not shown) were all small and nonsignificant; this suggests that the categories "most preferred," "acceptable," and "unacceptable" respect an underlying perceptual order.

That an underlying order exists is further borne out by examination of the canonical regression coefficients for the criterion set (normalized for comparison across facets). As noted from table 1, in all six cases "most preferred" carries a higher scale value than "acceptable," which in turn shows a higher scale value than "unacceptable." ("Unacceptable" carries an implied scale value of zero.) Moreover, we note that the scale value of "acceptable" tends to be relatively close to that of "unacceptable" in four out of the six facets. In no case is the scale value of "acceptable" at or above the midpoint between "most preferred" (1.0) and "unacceptable" (0.0).

\section{Constant Sum Results}

Figure 4 shows the average facet importance from phase 2's constant sum task. We note that room is highest in importance, followed by atmosphere/facilities and security. The more peripheral amenitiesassociated services, recreation/sports, and lounge-do not receive much importance.

\section{Part Worth Results}

Illustratively, table 2 shows the part worths (i.e., canonical regression coefficients), for the first facet: atmosphere/facilities. As noted, in some cases, such as hotel size, landscaping, and pool type, respondents appear to be avoiding the most expensive options in which the enhancement does not appear to be worth the money. However, in the 
Relative Importance of the Six Facets

Room Atmosphere/
facilities

Security

Associated services

Recreation/ sports

Lounge
$17.2 \%$

$17.2 \%$

Fig. 4.-Constant sum evaluation of the importance of the six facets

case of corridor/view and building shape, the additional premium appears to be more than justified by the amenity's attractiveness.

Similar analyses were made of the remaining five facets and a utility function incorporating the individual part worths, and facet importances were obtained for each respondent according to the hybrid procedure described earlier.

\section{Preference for Bundled Stimuli and Control for Blocking Effect}

A model analogous to equation (3) was first fitted to the phase 3 bundled data. As noted, the criterion variable is a 1-10 rating on a subjective likelihood-of-staying scale and the main predictor variable is the self-explicated part worth for the full hotel description (as illustrated in fig. 3). Since each repondent received a block of only five (out of 50) profiles, a nine dummy variables were added as predictors to measure any possible block effects. (The tenth block received a reference dummy coding of all zeros.)

As noted from table 3, only the regression coefficient associated with the self-explicated utility is significant at the .001 level. Thus, use of a balanced blocking factor (with all levels appearing an equal number of times within each block of five profiles) was successful in the sense that no block effect was apparent. 
TABLE 2 Part Worths for Attribute Levels within the Atmosphere/Facilities Facet

\begin{tabular}{|c|c|c|c|}
\hline $\begin{array}{l}\text { Attribute and } \\
\text { Levels }\end{array}$ & Description & $\begin{array}{c}\text { Price } \\
\text { Premium }\end{array}$ & $\begin{array}{l}\text { Part } \\
\text { Worth }\end{array}$ \\
\hline \multicolumn{4}{|l|}{ Hotel size: } \\
\hline 1 & Small (125 rooms) 2-story hotel & .00 & 1.06 \\
\hline & 12-story (600 rooms) with large & & \\
\hline & lobby, meeting rooms, etc. & 7.15 & .00 \\
\hline \multicolumn{4}{|l|}{ Corridor/view: } \\
\hline 1 & $\begin{array}{l}\text { Outside stairs and walkways to } \\
\text { all rooms; restricted view; peo- } \\
\text { ple walking outside window }\end{array}$ & .00 & .00 \\
\hline 2 & $\begin{array}{l}\text { Enclosed central corridors and } \\
\text { stairs; unrestricted view; } \\
\text { rooms have balcony or large } \\
\text { window }\end{array}$ & .65 & 1.85 \\
\hline \multicolumn{4}{|l|}{ Pool location } \\
\hline 1 & Not in courtyard & .00 & .00 \\
\hline 2 & In courtyard & .00 & 1.37 \\
\hline \multicolumn{4}{|l|}{ Pool type } \\
\hline 1 & No pool & .00 & .61 \\
\hline 2 & Rectangular pool & .45 & 1.25 \\
\hline 3 & Freeform pool & .50 & .29 \\
\hline \multirow{2}{*}{\multicolumn{4}{|c|}{$\begin{array}{l}4 \\
\text { Landscaping }\end{array}$}} \\
\hline & & & \\
\hline 1 & Minimal landscaping & .00 & .81 \\
\hline 2 & Moderate lanscaping & .10 & .97 \\
\hline 3 & Elaborate landscaping & .50 & .00 \\
\hline \multicolumn{4}{|l|}{ Building shape } \\
\hline 1 & $\begin{array}{l}\text { L-shaped building with modest } \\
\text { landscaping }\end{array}$ & .00 & .00 \\
\hline 2 & $\begin{array}{l}\text { Building forms an outdoor land- } \\
\text { scaped courtyard for sitting, } \\
\text { eating, sunning, etc. }\end{array}$ & .45 & .37 \\
\hline
\end{tabular}

\section{Assessing the Effect of the Overall Bundle Price}

The next step was to compute residuals from the regression summarized in table 3. These residuals were then regressed on the totallodging price variable, dummy coded according to the scheme shown in the unnumbered table below.

\begin{tabular}{lrc}
\hline & Price Range & Dummy Coding \\
\hline Lowest price & $\$ 27.75-31.75$ & $1,0,0,0$ \\
Low price & $31.76-35.75$ & $0,1,0,0$ \\
Intermediate price & $35.76-39.75$ & $0,0,1,0$ \\
High price & $39.76-46.75$ & $0,0,0,1$ \\
Highest price & $46.76-59.75$ & $0,0,0,0$ \\
\hline
\end{tabular}

As noted, the highest price range received the reference coding of all zeros.

Table 4 and figure 5 show the results. All of the price dummies are significant at the .05 level or better. Moreover, all the regression 
TABLE 3 Regression Results of Phase 3 Analysis

\begin{tabular}{lccc}
\hline Predictor Variable & Regression Weight & $t$-Value & Beta Weight \\
\hline Self-explicated utility & .030 & $4.574^{*}$ & .153 \\
Block 1 & .605 & 1.473 & .066 \\
Block 2 & .518 & 1.279 & .057 \\
Block 3 & .427 & .983 & .043 \\
Block 4 & .685 & 1.689 & .076 \\
Block 5 & .172 & .395 & .020 \\
Block 6 & .513 & 1.077 & .045 \\
Block 7 & .475 & 1.145 & .040 \\
Block 8 & .430 & 1.094 & .046 \\
Block 9 & .632 & 1.581 & .073 \\
Intercept & 2.359 & & \\
\hline
\end{tabular}

*Significant at the .001 level.

weights are (appropriately) positive and the "demand" curve is downward sloping. We conclude that the self-explicated utilities (see table 3 results) do not fully account for respondents' reactions to the full profiles appearing in phase 3.

\section{Cross-Validation}

While the regression coefficients in tables 3 and 4 are encouraging with respect to algebraic sign and statistical significance, the main question of interest is how well they predict individual-respondent evaluations. Accordingly, a leave-one-out procedure (Fenwick 1979) was set up in which each individual's first choice (among the five profiles evaluated) and his or her ranking of the five profiles (ties permitted) was predicted based on parameters computed for the rest of the sample. In short, each respondent's data were held out and predicted, one respondent at a time. ${ }^{4}$

The leave-one-out procedure indicated that 65 out of 180 respondents' first choices $(36.1 \%)$ were predicted. The average Spearman rank correlation was .253 for the full ranking of five profiles. While this

TABLE 4 Regression Results of Phase 3 Residuals Analysis

\begin{tabular}{lccc}
\hline Predictor Variable & Regression Weight & $t$-Value & Beta Weight \\
\hline Lowest price & 2.994 & $11.350^{*}$ & .447 \\
Low price & 2.148 & $7.899^{*}$ & .306 \\
Intermediate price & 1.260 & $4.687^{*}$ & .183 \\
High price & .514 & $1.963^{* *}$ & .078 \\
Intercept & -1.384 & & \\
\hline
\end{tabular}

\footnotetext{
* Significant at the .001 level.
}

**Significant at the .05 level.

4. The leave-one-out procedure was confined to the two regression equations, summarized in tables 3 and 4 . That is, the parameters obtained from the canonical correlations were not jackknifed due to prohibitive computer costs. 
The Price Utility Function for Hotel Amenities

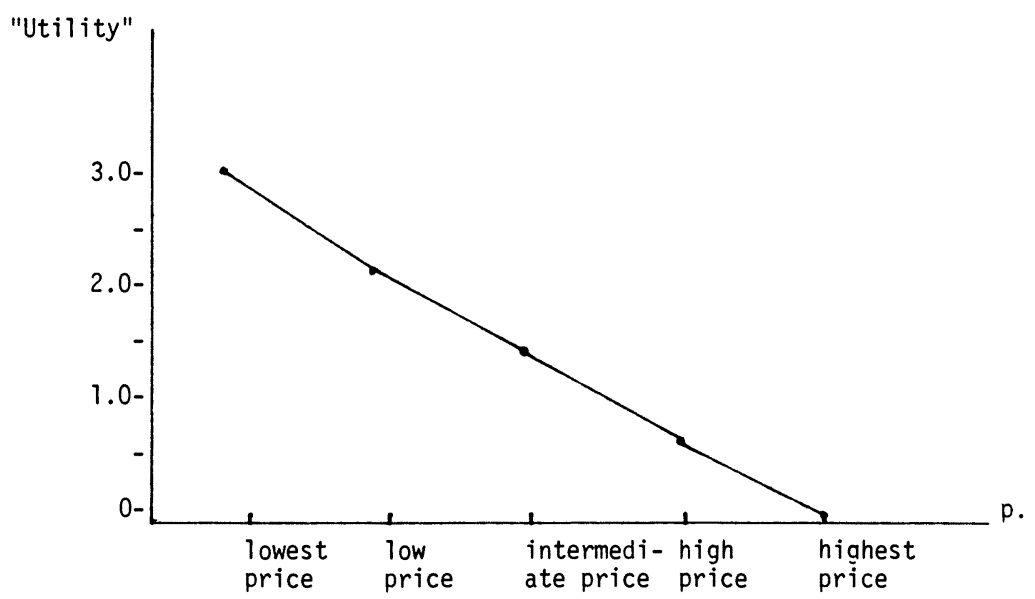

Fig. 5.-The price utility function for hotel amenities

is far from outstanding predictive performance, the results are encouraging, given the complexity of bundles (phase 3 data).

\section{The Case for Equal Facet Weights}

A second complete analysis was carried out in which equal facet weights were assumed. In this case the regression weights associated with the self-explicated utility and the price variables (tables 3 and 4) were quite similar to the cases in which self-explicated facet weights were used. Rather surprisingly, the leave-one-out predictions were somewhat better for the equal weights case -75 out of 180 first-choice predictions $(41.7 \%)$ and an average Spearman rank correlation of $.299 .^{5}$

We do not think that too much should be concluded about the robustness of facet weights from this single example. About all that can be said is that no severe degradation was noted in moving from selfexplicated to equal facet weighting.

\section{An Individual Differences Part-Worth Model}

In the hybrid models described above, the within-facet part worths, analogous to the $u_{i_{j}}$ 's of equation (1), were all estimated at the group level and then normalized to range between 0 and 10. Only the importance weights of phase 2 reflected individual variation. However, one might want to consider other hybrid models that reflect additional sources of individual variation.

5. A $\chi^{2}$ test was run on the differences between hit frequencies (self-explicated vs. equal facet weights) and a $t$-test was run on the differences between average Spearman correlations (converted to Fisher's Z). Neither test showed a significant difference at the $.05 \alpha$ level. Hence, the self-explicated and equal weights cases produced pretty much the same level of cross-validation. 
Consider table 1's normalized scale values for the response "'acceptable." As described earlier, most preferred can be coded 1.0 and unacceptable coded 0.0 . If we assume that these numerical values reflect appropriate spacing along a common preference scale, we can compute individualized part worths for each respondent. All we have to do is find each respondent's profile of responses (most preferred, acceptable, and unacceptable) and numerically encode them for the various attribute levels considered in each facet. Then, the $U_{i}, i_{2} \ldots i_{J *}$ 's of equation (3) will reflect differences in both phase 1 attribute-level desirabilities and phase 2 importance weights. This is analogous to fitting a metric hybrid model that allows for across-facet variation in the desirability value given to the "acceptable" response. Because different respondents can value the attribute levels differently, their self-explicated utilities can also differ.

Accordingly, this model was fitted to the data and cross-validated via a leave-one-out procedure. We found that 69 out of 180 respondents' first choices $(38.3 \%$ ) were correctly predicted; the Spearman rank correlation was .287 . When equal facet weights were used, 68 out of 180 first choices were correct $(37.8 \%)$ and the Spearman rank correlation was .245. From a practical standpoint, all four models produce similar cross-validated results, suggesting that the earlier use of group-level part worths (as illustrated in table 2) did not dramatically reduce the model's predictive accuracy, at least in terms of the leave-one-out cross-validation procedure. ${ }^{6}$

\section{Discussion}

We first summarize the study's substantive findings. Since this is the first application of the categorical hybrid conjoint model, the advantages and limitations of this methodology are discussed and some future applications of this methodology are suggested.

\section{The Substantive Findings}

The study results suggest the following conclusions (the findings are limited, of course, to the context and sample of this specific application).

1. Simple functions of respondents' self-explicated utilities for bundle components are not good predictors of their preferences for the total bundle of hotel amenities.

2. The overall bundle price adds significantly to the accounted-for variance in preference for hotel bundles.

3. As expected, the price-preference function is a downward-sloping demand curve (fig. 5).

6. Had individual importance weights for attributes within facets also been obtained, the individual differences models of this section could be expanded to include this source of individual variation as well. 
4. Individual respondent evaluations of the bundled stimuli can be predicted (from the parameters of the other respondents) in slightly over one-third of all cases-a relatively weak predictive validity.

5. The hybrid categorical conjoint analysis model produced (in this extremely large and complex real world application) "reasonable" results which, together with other information from this study, provided management with specific guidelines for the development of a new hotel chain.

6. In this specific case, the respondent-obtained importance weights did not provide greater predictive validity than simple (equal) weights.

One of the more interesting results was an empirical demonstration of Tversky's comments regarding the cumulative impact of small changes in price on preference "reversal." More research needs to be done on this problem. For example, is it possible that relatively high evaluations of enhancements with incremental price increases are reported because subjects feel that other enhancements will probably come along as well (without consideration of their price premiums)?

\section{Advantages and Limitations of Categorical Hybrid Models}

The major advantage of the categorical hybrid conjoint model is its computational speed and ease of application. In principle, one could construct a version of MONANOVA (Kruskal 1965) that imposes monotonicity on the three-point response scale and that is capable of handling missing data. However, as is well known, MONANOva is subject to local optima problems and solution degeneracy. It is also relatively expensive to run.

In contrast, the categorical hybrid model uses standard canonical correlation programs (e.g., P6M in the BMDP series) that can handle large-scale problems. Moreover, one does not need to impose monotonicity on the criterion (response) variable. This feature is especially important in those cases in which the response is truly categorical, such as choosing specific occasions for which a particular hotel might be appropriate. Of course, if the second set of linear compounds had turned out to yield large and significant canonical correlations, doubt is cast on the appropriateness of the model. In this case, the researcher might have to fall back on the imposition of monotonicity via MONANOVA or some similar procedure.

Three additional problems with the approach should be pointed out. First, in some problems, use of a three-category response scale might be questioned as too gross a classification. If so, the categorical hybrid model could still be used, although one would need to add more dummy variables in the criterion set-up to $K-1$ dummies for a $K$ category response. Indeed, one could experiment with a collapsing of response categories to see how coarse the gradations can be made and still be supported by the data.

Second, the present problem surely taxes the information absorption 
powers of the respondent in phase 3. (This is the largest conjoint problem which the authors have ever seen.) It is highly likely that the lack of strong cross-validation results reflects the high information overload in phase 3 .

Third, in phase 3 the present application permitted some correlation among attributes within facet but assumed orthogonality across facets in the construction of the 50 stimulus profiles. This constraint could be relaxed in future studies (with a resultant reduction in precision of estimation and greater ambiguity in the measurement of part worths).

\section{Other Categorical Hybrid Models}

We have illustrated only one version of the categorical hybrid model. Cases could arise in which separate regression weights might be desired for each facet, that is, where the model summarized in table 3 is modified to include a separate regression coefficient for each facet. These could be employed in lieu of (or in addition to) the selfexplicated importance weights obtained from phase 2 data.

Similarly, the model summarized in table 4 could include attributes in addition to price (e.g., situation descriptions, distance from airport) that the researcher wishes to parameterize on the basis of the fullprofile stage alone.

\section{Future Applications}

A large number of products and services-cars, boats, electrical appliances, single homes, condominiums, stereo and video equipment, computer terminals, copy machines, word processors, financial services-are often sold in terms of a basic unit and various add-ons that are optional at extra cost. The method described here (or some variation of it) may be applicable to this wide class of problems. It is hoped that additional studies, of the type illustrated here, might be undertaken. Moreover, the related problem of cue consistency and procedures for dealing with environmentally correlated attributes deserves attention in future studies in the general problem area of pricing research (Rao 1984).

\section{References}

Adams, W. J., and Yellen, J. L. 1976. Commodity bundling and the burden of monopoly. Quarterly Journal of Economics 90 (August): 475-98.

Addelman, Sidney. 1962. Symmetrical and asymmetrical fractional factorial plans. Technometrics 4 (February): 47-58.

Anderson, Norman H., and Jacobson, Ann. 1965. Effect of stimulus inconsistency and discounting instructions in personality impression formation. Journal of Personality and Social Psychology 2:531-39.

Carroll, J. Douglas. 1969. Categorical conjoint measurement. Paper presented at the annual meeting of mathematical psychology, University of Michigan, August.

Carroll, J. Douglas. 1973. Categorical conjoint measurement. In P. E. Green and Y. Wind (eds.), Multiattribute Decisions in Marketing. Hinsdale, Ill.: Dryden. 
Cattin, Philippe, and Wittink, Dick R. 1982. Commercial use of conjoint analysis: A survey. Journal of Marketing 46 (Summer): 44-53.

Dawes, Robyn M., and Corrigan, Bernard. 1974. Linear models in decision making. Psychological Bulletin 81:97-106.

Dudycha, Arthur L., and Naylor, James C. 1966. The effect of variations in the cue R matrix upon the obtained policy equation of judges. Educational and Psychological Measurement 26:583-603.

Edwards, Ward. 1976. Comment on equal weighting in multi-attribute models: A rationale, an example, and some extensions. In M. Schiff and G. Sorter (eds.), Proceedings of the Conference on Topical Research in Accounting. New York: New York University Press.

Einhorn, Hillel J., and McCoach, William. 1977. A simple multiattribute utility procedure for evaluations. Behavioral Science 22:270-82.

Fenwick, Ian. 1979. Techniques in market measurement: The jacknife. Journal of Marketing Research 16 (August): 410-14.

Green, Paul E.; Carroll, J. Douglas; and Carmone, Frank J. 1978. Some new types of fractional factorial designs for marketing experiments. In J. N. Sheth (ed.), Research in Marketing. Vol. 1. Greenwich, Conn.: JAI.

Green, Paul E., and Goldberg, Stephen M. 1981. A nonmetric version of the hybrid conjoint analysis model. Paper presented at the third ORSA/TIMS Market Measurement Conference, New York University, March.

Green, Paul E.; Goldberg, Stephen M.; and Montemayor, Mila. 1981. A hybrid utility estimation model for conjoint analysis. Journal of Marketing 45 (Winter): 33-41.

Green, Paul E.; Goldberg, Stephen M.; and Wiley, James B. 1982. A cross-validation test of hybrid conjoint models. Proceedings of the 1982 Annual Meeting of the Association for Consumer Research, San Francisco, October.

Green, Paul E., and Rao, Vithala R. 1971. Conjoint measurement for quantifying judgmental data. Journal of Marketing Research 8:355-63.

Green, Paul E., and Srinivasan, V. 1978. Conjoint analysis in consumer research: Issues and outlook. Journal of Consumer Research 5 (September): 103-23.

Hoffman, Paul J. 1968. Cue-consistency and configurality in human judgment. In B. Kleinmuntz (ed.), Formal Representation of Human Judgment. New York: Wiley.

Johnson, Richard M. 1974. Trade-off analysis of consumer values. Journal of Marketing Research 11:121-27.

Kruskal, Joseph B. 1965. Analysis of factorial experiments by estimating monotone transformations of the data. Journal of the Royal Statistical Society, ser. B, 27:251-63.

Parker, Barnett R., and Srinivasan, V. 1976. A consumer preference approach to the planning of rural primary health care facilities. Operations Research 24:991-1025.

Paroush, J., and Peles, Y. C. 1981. A combined monopoly and optimal packaging model. European Economic Review 15 (March): 373-83.

Phillips, O. R. 1981. Product bundles, price discrimination and the two product firm. Working paper. College Station: Texas A\&M University.

Rao, Vithala R. 1984. A review of pricing research in marketing. Journal of Business. In this issue.

Schmalensee, R. 1984. Gaussian demand and commodity bundling. Journal of Business. In this issue.

Shocker, Allan D., and Srinivasan, V., 1977. LinmaP (Version II): A ForTRAN IV computer program for analyzing ordinal preference (dominance) judgments via linear programming techniques and for conjoint measurement. Journal of Marketing Research 14:101-3.

Slovic, Paul. 1966. Cue-consistency and cue-utilization in judgment. American Journal of Psychology 79:427-34.

Spence, A. M. 1980. Multi-product quantity-dependent priced and profitability constraints. Review of Economic Studies 47 (October): 821-42.

Stigler, G. J. 1968. A note on block booking reprinted in The Organization of Industry, by C. J. Stigler. Homewood, Ill.: Irwin.

Telser, L. G. 1979. A theory of monopoly of complementary goods. Journal of Business 52 (April): 211-30.

Tversky, Amos. 1969. Intransitivity of preferences. Psychological Bulletin 76:31-48.

Wilkie, William, and Pessemier, Edgar A. 1973. Issues in marketing's use of multiattribute attitude models. Journal of Marketing Research 10 (November): 428-41. 